\title{
Multicomponent Fuel Diffusion Flames: Flame Structure for Coupled Diffusion-Flame and Premixed-Flame Burning Regimes*
}

\author{
Fernando F. Fachini \\ ${ }^{\dagger}$ Instituto Nacional de Pesquisas Espaciais-INPE
}

\begin{abstract}
This analysis addresses some aspects of the extinction and the structure of diffusion flames established by burning of two fuels. This analysis is asymptotically performed presuming one-global-step chemical reactions with large activation energies. By working with fuels with different activation energies, the structure is composed by two zones with different thickness. The reactions of the fuels with oxygen occur practically inside a determined zone. The reaction with the highest activation energy takes place in a thin zone and that one with the smallest activation energy takes place in a thick zone. The results pointed out that the structure of the thick zone is of the diffusion flame. However, the structure of the thin zone is of the lean-premixed-flame. Also, it was found that the thin zone is inside the thick zone and its position depends on the value of the Damköhler number of the reaction that occurs in the thin zone. Under the conditions followed by this analysis the reactions zones form a single flame.
\end{abstract}

\section{Introduction}

$D^{n}$

ESPITE multicomponent fuels are employed in an extensive application range from electricity generation to air transportation, not enough attention has been spent in order to increase the combustion process understanding of these fuels. In this connection, the aim of this analysis is to investigate the diffusion flame structure established by the multicomponent fuels burning.

This work is an extension of the previous multicomponent-fuel diffusion flames analyses. ${ }^{1,2}$ Hamins and Seshadri ${ }^{1}$ analyzed theoretically and experimentally the binary and ternary liquid fuels (toluene, n-heptane and methanol) mixtures extinction which were burned in a pool configuration with air stream being directed against the liquid surface. The chemical-kinetic parameters (i.e. activation energy and frequency factor) employed in that analysis were determined experimentally in the flame extinction conditions, for each fuel in an isolated way. ${ }^{3}$ In order to determine an analytical solution for the flame extinction condition, in that analysis they considered adiabatic condition in one side of the flame. Even considering this assumption, the results dictated by the large activation energy asymptotics showed good agreement with the experimental

\footnotetext{
*43rd AIAA Aerospace Sciences Meeting and Exhibit, 10-13 January, Reno, Paper AIAA 2005-0550

${ }^{\dagger}$ Cachoeira Paulista, São Paulo, 12630-000,Brazil
} 
results. ${ }^{1}$ In addition, they developed a mixing rule to determine the overall chemical-kinetic parameters that represent the fuels mixture as one hypothetical fuel. ${ }^{4,5}$ Moreover, other experimental study evolving mixtures of the same three liquid fuels showed that the consumption of the toluene and n-heptane occurs at the same region inside the flame, however the consumption of methanol does not occur in the same region ${ }^{6}$.

Employed binary gaseous mixture of n-heptane and hydrogen, Fachini ${ }^{2}$ extended Hamins and Seshadri's ${ }^{1}$ theoretical analysis relaxing the adiabatic condition and assuming Lewis number equal to one. The analysis showed that the extinction condition is controlled by the reaction with the largest activation energy and smallest Damköhler number. In addition, the analysis revealed that the extinction conditions do not change significantly when a Damköhler number of one reaction is much larger than the Damköhler numbers of the other reactions. The same behavior for the extinction conditions was observed when the activation energy of one reaction is less than one half of the others activation energy.

The main objetive of this work is of extending the Fachini's analysis ${ }^{2}$ employing the activation energy and Damköhler number of one reaction much larger than those of other reactions. Due to the large difference among activation energies, the reaction with the largest activation energy occurs in a zone much thinner than that of other reactions. In this analysis, the Damköhler number of the largest activation-energy reaction will be chosen such that through the thin reaction zone the leakage of fuel is proportional to the inverse of the largest activation energy, but the leakage of the oxygen will be of the same order of the concentrations of the species in the thick reaction zone. Under these conditions, the fuel burning inside the thin zone occurs in premixed-flame regime. ${ }^{7}$ Thus, the structure of the thin reaction zone, imposed by the Damköhler number, is stable only inside the thick reaction zone. In addition, the oxygen not consumed at the thin reaction zone sustains the reactions in the other part of the thick reaction zone.

The thick reaction zone is almost similar to that described in previous analyses, ${ }^{1,2}$ but with a discontinuous oxygen flux across the thin reaction zone. That zone is unlimited in both sides and fuel and oxygen leakages are of the order of inverse of the activation energy. Under this condition, the fuel burns inside the thick reaction zone in diffusion-flame regime. ${ }^{7}$

\section{Flow Field Description}

The asymptotical flame structure description depends directly on flame properties. The flow field influence is secondary. To provide these properties a counterflow diffusion flame is analysed. From the left stream of the counterflow, air is injected into the domain and from the right stream, a mixture of n-heptane and hydrogen is injected. The length of the domain is $l$ and the axial coordinate origin is set on the oxygen stream, $z=0$, then fuel stream is at $z=l$. In the model, the radiative energy transfer is included by the approximation of optically thin transparent gas and released in the $\mathrm{CO}_{2}$ and $\mathrm{H}_{2} \mathrm{O}$ bands. ${ }^{8,9,10,11}$ This

2 of 21

American Institute of Aeronautics and Astronautics 
model considered that the thermal conductivity $k$, diffusion coefficient $D$, coefficient of viscosity $\mu$ depend only on nondimensional temperature $\theta=T / T_{2}$,

$$
\frac{\rho D_{i}}{\rho_{2} D_{i 2}}=\frac{k}{k_{2}}=\frac{\mu}{\mu_{2}}=\theta^{\alpha}
$$

The specific heat at constant pressure $c_{p}$ is constant. The subscript 1 corresponds to the condition at the air stream and the subscript 2 corresponds to the condition at the fuel stream.

This work considers the multicomponent fuel composed by two species. In terms of mass, the chemical reactions of a general multicomponent fuel proceed at one global step according to

$$
F_{i}+s_{i} O_{2} \rightarrow \tilde{c}_{i} \mathrm{CO}_{2}+\tilde{h}_{i} \mathrm{H}_{2} \mathrm{O} \quad+Q_{i}
$$

in which $s_{i}, \tilde{c}_{i}$ and $\tilde{h}_{i}$ are the massic stoichiometric coefficients of reaction $i$, i.e., for each unit mass of fuel i, $s_{i}$ oxygen mass is consumed to have stoichiometric reaction; with the multicomponent fuel composed by $n$ species, $i=1, \ldots, n$. These coefficients are expressed by $s_{i}=n_{O_{2}, i} M_{O_{2}} / M_{F_{i}}, \tilde{c}_{i}=n_{C O_{2}, i} M_{C O_{2}} / M_{F_{i}}$ and $\tilde{h}_{i}=n_{\mathrm{H}_{2} \mathrm{O}, i} M_{\mathrm{H}_{2} \mathrm{O}} / M_{\mathrm{F}_{i}}$, in which $n_{\mathrm{O}_{2}, i}, n_{\mathrm{CO}_{2}, i}, n_{\mathrm{H}_{2} \mathrm{O}, i}, M_{\mathrm{O}_{2}}, M_{\mathrm{CO}_{2}}$ and,$M_{\mathrm{H}_{2} \mathrm{O}}$ stand as molar stoichiometric coefficients for reaction $i$ and the molar weight for $\mathrm{O}_{2}, \mathrm{CO}_{2}, \mathrm{H}_{2} \mathrm{O} . \mathrm{M}_{\mathrm{F}_{i}}$ is the molar weight for the fuel $i$. Note that $1+s_{i}=\tilde{c}_{i}+\tilde{h}_{i}$. $Q_{i}$ is the heat released by the reaction $i$.

The nondimensional rates of the chemical reactions are expressed by

$$
w_{i}=D a_{i} \varrho^{\beta_{i 1}+\beta_{i 2}} y_{O}^{\beta_{i 1}} y_{i}^{\beta_{i 2}} e^{-\theta_{a i} / \theta}, \quad i=1,2
$$

where $\beta_{i}$ and $\theta_{a i}=E_{i} / R T_{2}$ are the global reaction order and the nondimensional activation energy of reaction $i$, respectively. The definitions for the re-scaled oxygen and fuels mass fractions, $y_{O}$ and $y_{i}$, are as following

$$
y_{O}=Y_{O} / Y_{O 1}, \quad y_{i}=s_{i} L e_{O} Y_{i} /\left(Y_{O 1} L e_{i}\right)
$$

The Damköhler number $D a_{i}$ is defined as

$$
D a_{i}=\operatorname{Pe}\left(B_{i} l / v_{2}\right)\left(\rho_{2} Y_{O 1}\right)^{\beta_{i 1}+\beta_{i 2}-1} L e_{i}^{\beta_{i 2}} /\left(s_{i} L e_{O}\right)^{\beta_{i 2}-1}
$$

$B_{i}, v, \rho, L e_{i}=D_{i 2} /\left(k_{2} / \rho_{2} c_{p}\right)$ and $P e=l v_{2} /\left(k_{2} / \rho_{2} c_{p}\right)$ are the frequency factor, the velocity of the streams, the density, the Lewis number of fuel $i$ and the Peclet number, respectively.

The flow field properties used in the flame structure analysis, flame position, such as flame temperature, the heat flux from the flame and the oxygen and fuels fluxes to the flame, are as following

$$
\begin{gathered}
x=x_{f}, \quad \theta=\theta_{f}, \quad d_{\theta}^{-}=\left.\frac{d \theta}{d x}\right|_{x=x_{f}^{-}}, \quad d_{\theta}^{+}=-\left.\frac{d \theta}{d x}\right|_{x=x_{f}^{+}}, \\
d_{0}=-\left.\frac{d y_{O}}{d x}\right|_{x=x_{f}}, \quad d_{i}=\left.\frac{d y_{i}}{d x}\right|_{x=x_{f}}
\end{gathered}
$$

3 of 21

American Institute of Aeronautics and Astronautics 
respectively. The nondimensional axial coordinate is $x=z / l$, thus the oxygen stream starts at $x=0$ and the fuel stream at $x=1$. According to the definition of $y_{0}, y_{i}(i=1 \ldots n), d_{0}, d_{i}(i=1 \ldots n)$ and the stoichiometric condition for the reactants at the flame, the following relation $d_{0}+\sum_{i=1}^{n} d_{i}=0$ is satisfied.

More details about the counterflow description considering multicomponent fuel can be found anywherelse. $^{12}$

\section{Flame Structure}

As considered in the leading order problem describing either one-fuel diffusion flames ${ }^{13}$ or multicomponent fuel diffusion flames, ${ }^{12}$ the chemical reaction was performed infinitely fast, thereby it occurs in an infinitely thin zone; there is no leakage of fuel and oxygen through the flame. This assumption makes the derivatives of the variables discontinuous at the flame. To eliminate that discontinuity, it is necessary to solve the problem for the next approximation that corresponds to the description of the reactants consumption inside the flame. Defining the hydrogen as fuel 1 and n-heptane as fuel 2, in

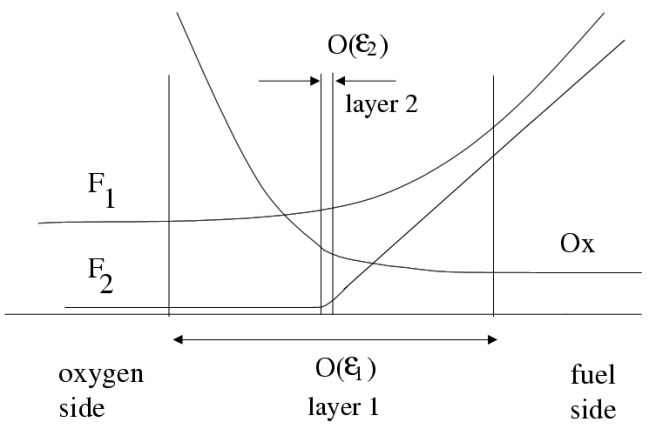

Figure 1. Schematic representation of the internal structure of a diffusion flame through the profiles of fuels 1 and 2 and of oxygen.

the limit $\theta_{a 2} \gg \theta_{a 1} \gg 1$ presumed in this work, the flame presents a different internal structure. Again, in the leading order problem, the flame is still considered infinitely thin. However, in this work, at order of magnitude corresponding proportionally to $1 / \theta_{a 1}$ the problem, which describes the consumption of hydrogen (fuel 1), treats the zone where n-heptane (fuel 2) is consumed as infinitely thin. The reason for that is because the reaction 2 is much more dependent on temperature than the reaction 1 . Since both reactions compete for oxygen, it is reasonable to expect that the zone where n-heptane (fuel 2) is consumed is inside the zone where hydrogen (fuel 1) is consumed. The zone where hydrogen (fuel 1) is consumed is called layer 1 and the zone where n-heptane (fuel 2) is consumed is called layer 2.

The position of the layer 2 inside the layer 1 is determined by the physical and chemical properties of the problem. In this work, the properties were chosen in a such way that, the layer 2 is inside layer 1 , as seen in figure 1. With this configuration, the flame is less stable because the leakage of oxygen from the thin layer 2 is of the order of $1 / \theta_{a 1}$, much larger than $1 / \theta_{a 2}$ that is the (fuel 2) n-heptane leakage. Therefore, the flame configuration is formed by the hydrogen reaction zone controlled by the diffusion-flame regime and the n-heptane reaction zone controlled by the premixed-flame regime, according to definition. ${ }^{7}$ 
To study a bicomponent fuel diffusion flame with distinct activation energies the following expansion must be considered

$$
\begin{aligned}
& \theta-\theta_{f}=-\varepsilon_{1}\left(\Theta_{1}+\gamma_{1} \xi_{1}\right) / \delta_{1}^{1 / b_{1}} \quad-\varepsilon_{2}\left(\Theta_{2}+\gamma_{2} \xi_{2}+p\right) \quad+\cdots \\
& y_{0}=\quad \varepsilon_{1}\left(d_{1}+d_{2}\right) \Psi_{01} / m_{1} \delta_{1}^{1 / b_{1}}+\varepsilon_{2} \Psi_{02}+\cdots \\
& y_{1}=\quad \varepsilon_{1} d_{1} \Psi_{11} / m_{1} \delta_{1}^{1 / b_{1}} \quad+\varepsilon_{2} d_{1} \Psi_{12} / m_{2} \quad+\cdots \\
& y_{2}=\quad \begin{array}{lll}
\varepsilon_{1} d_{2} \Psi_{21} / m_{1} \delta_{1}^{1 / b_{1}}+\varepsilon_{2} d_{2} \Psi_{22} \quad+\cdots \\
\varepsilon_{1} \xi_{1} / m_{1} \delta_{1} / b_{1}
\end{array} \\
& x-x_{f}=\quad \varepsilon_{1} \xi_{1} / m_{1} \delta_{1}^{1 / b_{1}}+\varepsilon_{2}\left(\xi_{2} / m_{2}+p / \gamma_{2}\right) \quad+\cdots
\end{aligned}
$$

where

$$
\begin{array}{ll}
b_{1}=1+\beta_{11}+\beta_{12}, & b_{2}=1+\beta_{21}+\beta_{22}, \\
\varepsilon_{1}=\theta_{f}^{2} / \theta_{a 1}, & \varepsilon_{2}=\theta_{f}^{* 2} / \theta_{a 2}=\left(\theta_{f}-\varepsilon_{1} \Theta_{1 f} / m_{1} \delta_{1}^{1 / b_{1}}\right)^{2} / \theta_{a 2}, \\
m_{1}=\left(d_{\theta}^{-}+d_{\theta}^{+}\right) / 2 & m_{2}=\left(d_{\theta}^{+*}-d_{\theta}^{-*}\right)=d_{2} q_{2} \\
\gamma_{1}=1-2 d_{\theta}^{-} /\left(d_{\theta}^{-}+d_{\theta}^{+}\right), & \gamma_{2}=d_{\theta}^{-*} / q_{2} d_{2}, \\
\delta_{1}=\frac{D a_{1} e^{-\theta_{a 1} / \theta_{f}}\left(d_{1}+d_{2}\right)^{\beta_{11}} d_{2}^{\beta_{12}-1}}{\theta_{f}^{\alpha+\beta_{11}+\beta_{12}}}\left(\frac{\varepsilon_{1}}{m_{1}}\right)^{1+\beta_{11}+\beta_{12}}\left(\frac{d_{1}}{d_{1}+d_{2}}\right)^{\beta_{11}},
\end{array}
$$

The definition of $d_{1}, d_{2}, d_{\theta}^{-}$and $d_{\theta}^{+}$were already given in the previous section and they represent the fluxes of $y_{1}$ and $y_{2}$ and the heat fluxes at the flame in the oxygen side and in the fuel side of the flame, respectively. It ca be advanced that, $d_{\theta}^{-*}$ is the temperature slope at the position of the layer 2 in the oxygen side.

\section{A. Solution of order $\varepsilon_{1}$}

Everywhere inside layer 1, the influence of the reaction of n-heptane (fuel 2) on the oxygen consumption and on heat released is very small compared to that of the hydrogen (fuel 1), except inside the layer 2 located at $\xi_{1}=\xi_{1 f}\left(z=z_{f}^{*}=z_{f}+\varepsilon_{1} \xi_{1 f} / m_{1} \delta_{1}^{1 / b_{1}}\right)$. Since the layer 2 is inside the layer 1, the n-heptane (fuel 2) reaction follows a lean premixed-flame configuration. This is because the n-heptane (fuel 2) mass fraction goes to zero at $\xi_{1}=\xi_{1 f}, \Psi_{21}=0$, and the oxygen mass fraction $\Psi_{01}$ has a value of the order of unit, $\Psi_{01}=O(1)$ at $\xi_{1}=\xi_{1 f}$, in the problem of the order of $\varepsilon_{1}$. Moreover, the reaction rate of the oxidation of n-heptane (fuel 2) can be represented by delta function for the approximation of the order $\varepsilon_{1}$ at $\xi_{1}=\xi_{1 f}$. As will be seen later $d \Psi_{21} /\left.d \xi_{1}\right|_{\xi_{1}=\xi_{1 f}^{-}}=0$ and $d \Psi_{21} /\left.d \xi_{1}\right|_{\xi_{1}=\xi_{1 f}^{+}}=1$, thereby the n-heptane (fuel 2) reaction can be expressed as $d_{2} \delta\left(\xi_{1}-\xi_{1 f}\right)$. Therefore, as occurred in the leading order problem, the problem of the order $\varepsilon_{1}$ also has discontinuous derivatives for the functions $\Theta_{1}$ and $\Psi_{01}$ and $\Psi_{21}$ at $\xi_{1}=\xi_{1 f}$. The jump condition in the first derivative of $\Theta_{1}, \Psi_{01}$, and $\Psi_{21}$ at the layer $2, \xi_{1}=\xi_{1 f}$, will be used in the analysis of the order $\varepsilon_{2}$.

The hydrogen (fuel 1) reaction occurs in the layer 1 , which has a thickness of the order $\varepsilon_{1}$. The variations of the temperature and the reactants in this layer are followed by the system of equations of the order $\varepsilon_{1}$,

5 of 21

American Institute of Aeronautics and Astronautics 
resulting of taking equation (1) into the balance equations, ${ }^{12}$

$$
\begin{aligned}
\frac{d^{2}}{d \xi_{1}^{2}} m_{1}\left(\Theta_{1}+\gamma_{1} \xi_{1}\right) & =q_{1} d_{1}\left(\frac{d_{1}+d_{2}}{d_{1}}\right)^{\beta_{11}} \Psi_{01}^{\beta_{11}} \Psi_{11}^{\beta_{12}} e^{-\left(\Theta_{1}+\gamma_{1} \xi_{1}\right) / \delta_{1}^{1 / b_{1}}}+q_{2} d_{2} \bar{w}_{2} \\
\frac{d^{2}}{d \xi_{1}^{2}}\left(d_{1}+d_{2}\right) \Psi_{01} & =d_{1}\left(\frac{d_{1}+d_{2}}{d_{1}}\right)^{\beta_{11}} \Psi_{01}^{\beta_{21}} \Psi_{11}^{\beta_{12}} e^{-\left(\Theta_{1}+\gamma_{1} \xi_{1}\right) / \delta_{1}^{1 / b_{1}}}+d_{2} \bar{w}_{2} \\
\frac{d^{2}}{d \xi_{1}^{2}} d_{2} \Psi_{11} & =d_{1}\left(\frac{d_{1}+d_{2}}{d_{1}}\right)^{\beta_{11}} \Psi_{01}^{\beta_{11}} \Psi_{21}^{\beta_{12}} e^{-\left(\Theta_{1}+\gamma_{1} \xi_{1}\right) / \delta_{1}^{1 / b_{1}}} \\
\frac{d^{2}}{d \xi_{1}^{2}} d_{2} \Psi_{21} & =d_{2} \bar{w}_{2}
\end{aligned}
$$

The reaction rate $\bar{w}_{2}$ is defined as

$$
\bar{w}_{2}=\Delta \Psi_{01}^{\beta_{21}} \Psi_{21}^{\beta_{22}} e^{-\left(\theta_{a 2} / \theta_{a 1}\right) \cdot\left(\Theta_{1}+\gamma_{1} \xi_{1}\right) / \delta_{1}^{1 / b_{1}}}
$$

where $\Delta$ is defined as

$$
\Delta=\left[\frac{D a_{1}}{\theta_{f}^{\alpha+\beta_{21}+\beta_{22}}}\left(\frac{\varepsilon_{1}}{m_{1} \delta_{1}^{1 / b_{1}}}\right)^{b_{2}}\left(d_{1}+d_{2}\right)^{\beta_{21}} d_{2}^{\beta_{22}-1} e^{-\theta_{a 1} / \theta_{f}}\right]\left(\frac{D a_{2}}{D a_{1}} \frac{e^{-\theta_{a 2} / \theta_{f}}}{e^{-\theta_{a 1} / \theta_{f}}}\right)
$$

Since the content inside the brackets is of the order unity by the definition of $\delta_{1}$, then the order of magnitude of the $\Delta$ is given by the term inside the parenthesis. In addition, the Damköhler number $D a_{2}$ is very large to make the term $\left[D a_{2} \exp \left(-\theta_{a 2} / \theta_{f}\right)\right] /\left[D a_{1} \exp \left(-\theta_{a 1} / \theta_{f}\right)\right]$ large too. However, the value of the $\Delta$ is not very large enough to make the chemical rate $\bar{w}_{2}, \sim \Delta \exp \left(-\theta_{a 2} / \theta_{a 1}\right)$, of the order of unity everywhere inside the layer 1 . The value of chemical rate $\bar{w}_{2}$ is important only at a very thin zone where layer 2 is placed, $\xi_{1}=\xi_{1 f}$. In $\varepsilon_{1}$ approximation, the reaction rate $\bar{w}_{2}$ can be expressed as delta function $\bar{w}_{2}=d_{2} \delta\left(\xi_{1}-\xi_{1 f}\right)$. Then, for the $\varepsilon_{1}$ approximation problem, the layer 2 is an infinitely thin surface. Together with this approximation, all information already described in the Burke-Schumann problem can be applied to the problem of order $\varepsilon_{1}$ : the n-heptane (fuel 2) mass fraction after this thin zone is zero and the first derivative of the other variables are discontinuous.

The boundary conditions of equations (2) to (5), determined by the matching with the leading order solutions in the oxygen side, are given by

$$
\frac{d \Theta_{1}}{d \xi_{1}}+1=\frac{d \Psi_{01}}{d \xi_{1}}-1=\frac{d \Psi_{11}}{d \xi_{1}}=\frac{d \Psi_{21}}{d \xi_{1}}=\Psi_{11}=0, \quad \xi_{1} \rightarrow-\infty
$$

From the matching with the leading order solutions in the fuel side, the following boundary conditions are determined

$$
\frac{d \Theta_{1}}{d \xi_{1}}-1=\frac{d \Psi_{01}}{d \xi_{1}}=\frac{d \Psi_{11}}{d \xi_{1}}-1=\frac{d \Psi_{21}}{d \xi_{1}}-1=0, \quad \xi_{1} \rightarrow \infty
$$

Note that the definitions of $m_{1}$ and $\gamma_{1}$ are such that permit the derivative of $\Theta_{1}$ to be normalized for $\xi_{1} \rightarrow-\infty$ and $\xi_{1} \rightarrow \infty$. 
The condition at the layer 2 determines the jump for the first derivative of the functions $\Theta_{1}$ and $\Psi_{01}$, and $\Psi_{21}$. By performing the integration of the system of equations (2) to (5) around $\xi_{1}=\xi_{1 f}$ leads to

$$
\left.\frac{d}{d \xi_{1}}\left[\frac{m_{1}}{d_{2} q_{2}}\left(\Theta_{1}+\gamma_{1} \xi_{1}\right)\right]\right|_{\xi_{1 f}^{-}} ^{\xi_{1 f}^{+}}=\left.\frac{d}{d \xi_{1}}\left[\frac{\left(d_{1}+d_{2}\right)}{d_{1}} \Psi_{01}\right]\right|_{\xi_{1 f}^{-}} ^{\xi_{1 f}^{+}}=\left.\frac{d}{d \xi_{1}} \Psi_{21}\right|_{\xi_{1 f}^{-}} ^{\xi_{1 f}^{+}}=1, \quad \Psi_{21}\left(\xi_{1}=\xi_{1 f}^{-}\right)=0
$$

From Eq. (8), the definitions of $d_{\theta}^{-*}$ and $d_{\theta}^{+*}$ are established according to

$$
d_{\theta}^{-*}=\left.\frac{d}{d \xi_{1}}\left[m_{1}\left(\Theta_{1}+\gamma_{1} \xi_{1}\right)\right]\right|_{\xi_{1 f}^{-}}, \quad d_{\theta}^{+*}=\left.\frac{d}{d \xi_{1}}\left[m_{1}\left(\Theta_{1}+\gamma_{1} \xi_{1}\right)\right]\right|_{\xi_{1 f}^{+}}
$$

The heat flux at $\xi_{1}=\xi_{1 f}$ can be related with the heat flux from the flame to the oxygen side (Appendix A).

By integrating Eq. (5) with the boundary conditions (6) and (7) one finds

$$
\Psi_{21}= \begin{cases}0, & \xi_{1}<\xi_{1 f} \\ \xi_{1}-\xi_{1 f}, & \xi_{1} \geq \xi_{1 f}\end{cases}
$$

Exploring the fact that the excess enthalpy function ${ }^{14,15,10}$ defined as $H=\left(q_{1}-1\right) y_{1}+\left(q_{2}-1\right) y_{2}+y_{0}+\theta$, and mixture fraction, defined as $Z=y_{1}+y_{2}-y_{0}+1$, and their first derivatives are continuous everywhere, it is possible to find expressions relating the variables $\Theta_{1}, \Psi_{01}, \Psi_{11}$ and $\Psi_{21}$ as following

$$
\begin{gathered}
\left(q_{1}-1\right) d_{1} \Psi_{11}+\left(q_{2}-1\right) d_{2} \Psi_{21}+\left(d_{1}+d_{2}\right) \Psi_{01}-m_{1}\left(\Theta_{1}+\gamma_{1} \xi_{1}\right)=d_{H} \xi_{1} \\
\left.d_{1} \Psi_{11}+d_{2} \Psi_{21}-\left(d_{1}+d_{2}\right) \Psi_{01}+\gamma_{1} \xi_{1}\right)=d_{Z} \xi_{1}
\end{gathered}
$$

where $d_{H}$ and $d_{Z}$ are the derivatives of the functions $H$ and $Z$ at the flame $z=z_{f}$ [1], which are

$$
\begin{gathered}
d_{H}=-\left(d_{1}+d_{2}\right)+d_{\theta}^{-}=\left(q_{1}-1\right) d_{1}+\left(q_{2}-1\right) d_{2}-d_{\theta}^{+} \\
d_{Z}=d_{1}+d_{2}
\end{gathered}
$$

Therefore, from Eqs. (9), (10) and (11) and the values of $d_{H}$ and $d_{Z}$ one finds relations between $\Psi_{11}$ and $\Theta_{1}$ and between $\Psi_{11}$ and $\Psi_{01}$ like these

$$
\begin{array}{ll}
\left(q_{1} d_{1} / m_{1}\right) \Psi_{11}-\Theta_{1}-\xi_{1}=0 & \xi_{1} \leq \xi_{1 f} \\
\left(q_{1} d_{1} / m_{1}\right) \Psi_{11}-\Theta_{1}-\xi_{1}=\left(q_{2} d_{2} / m_{1}\right)\left(\xi_{1 f}-\xi_{1}\right) & \xi_{1} \geq \xi_{1 f}
\end{array}
$$

and

$$
\begin{array}{ll}
{\left[d_{1} /\left(d_{1}+d_{2}\right)\right]\left[\left(\Psi_{11}-\xi_{1}\right)-\left(d_{2} / d_{1}\right) \xi_{1}\right]-\Psi_{01}=0} & \xi_{1} \leq \xi_{1 f} \\
{\left[d_{1} /\left(d_{1}+d_{2}\right)\right]\left[\left(\Psi_{11}-\xi_{1}\right)-\left(d_{2} / d_{1}\right) \xi_{1 f}\right]-\Psi_{01}=0} & \xi_{1} \geq \xi_{1 f}
\end{array}
$$

Eqs. (12) and (13) provide relations between the variables $\left(\Theta_{1}, \Psi_{11}\right)$ and $\left(\Psi_{01}, \Psi_{11}\right)$, which taken into Eqs. (2) to (5) reduce to one the number of differential equations of the problem. According to Eq. (8), the first derivative of the functions $\Psi_{01}$ and $\Theta_{1}$ is discontinuous at the layer $2, \xi_{1}=\xi_{1 f}$. Only the function $\Psi_{11}$ is not discontinuous at $\xi_{1}=\xi_{1 f}$, and it is also continuous even at the second derivative. For this reason, the

$$
7 \text { of } 21
$$


conservation equation of the function $\Psi_{11}$ is chosen to be integrated, satisfying the boundary conditions (6) and (7), to find the solution of the problem. Equation of the function $\Psi_{11}$ is given by as following

$$
\begin{gathered}
\frac{d^{2}}{d \xi_{1}^{2}} \Psi_{11}=\Psi_{11}^{\beta_{12}}\left\{\begin{array}{l}
{\left[\left(\Psi_{11}-\xi_{1}\right)-\left(d_{2} / d_{1}\right) \xi_{1}\right]^{\beta_{11}}} \\
{\left[\left(\Psi_{11}-\xi_{1}\right)-\left(d_{2} / d_{1}\right) \xi_{1 f}\right]^{\beta_{11}}}
\end{array} \times\right. \\
\exp \begin{cases}-\left[\Psi_{11}-d_{\theta}^{-} /\left(q_{1} d_{1}\right) \xi_{1}\right] /\left[\delta_{1} /\left(q_{1} d_{1} / m_{1}\right)^{b_{1}}\right]^{1 / b_{1}} & \text { for } \xi_{1} \leq \xi_{1 f} \\
-\left[\Psi_{11}-d_{\theta}^{-} /\left(q_{1} d_{1}\right) \xi_{1}+\left(q_{2} d_{2} / q_{1} d_{1}\right)\left(\xi_{1}-\xi_{1 f}\right)\right] /\left[\delta_{1} /\left(q_{1} d_{1} / m_{1}\right)^{b_{1}}\right]^{1 / b_{1}} & \text { for } \xi_{1} \geq \xi_{1 f}\end{cases}
\end{gathered}
$$

Note that, through the layer $2, \xi_{1}=\xi_{1 f}$, the temperature and oxygen mass fraction, $\Theta_{1}$ and $\Psi_{01}$, respectively, are continuous, then the second derivative of the hydrogen (fuel 1) mass fraction is also continuous. Also, note that for very low n-heptane (fuel 2) mass flux to the flame compared to the hydrogen (fuel 1) mass flux, the condition $d_{2} / d_{1} \ll 1$ is satisfied and Eq. (14) goes to the equation that describes the structure of a single fuel diffusion flame.

The position of layer $2, \xi_{1}=\xi_{1 f}$, is the eigenvalue of the problem that must be evaluated together with the solution of Eq. (14) that satisfies the previously Eqs. (6) and (7). However, there is no extra condition in the problem besides those two boundary conditions, which would permit to determine $\xi_{1 f}$. As occurred in the asymptotic description of premixed flames, an extra relation between the unknown flame properties with the position of layer 2 is obtained from the next approximation problem. Therefore, the problem corresponding to Eqs. (14), (6) and (7) is coupled to the problem that describes the structure of the layer 2, in the order of $\varepsilon_{2}$.

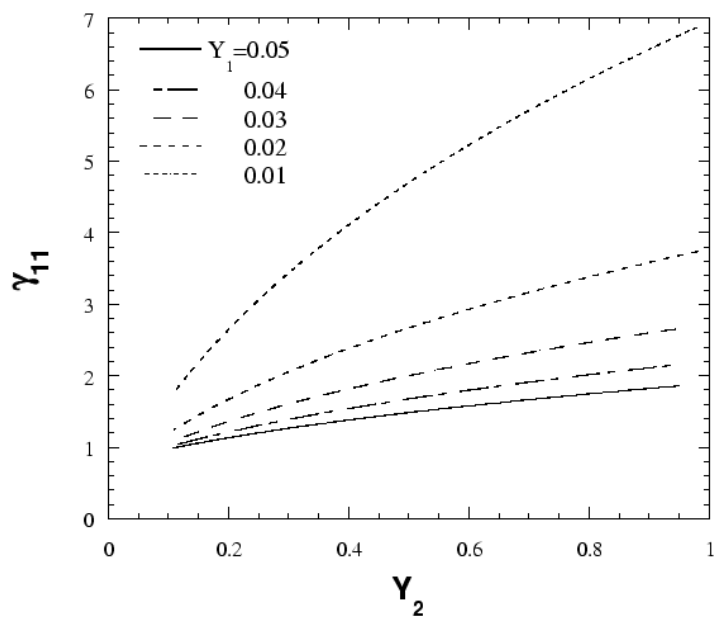

(a)

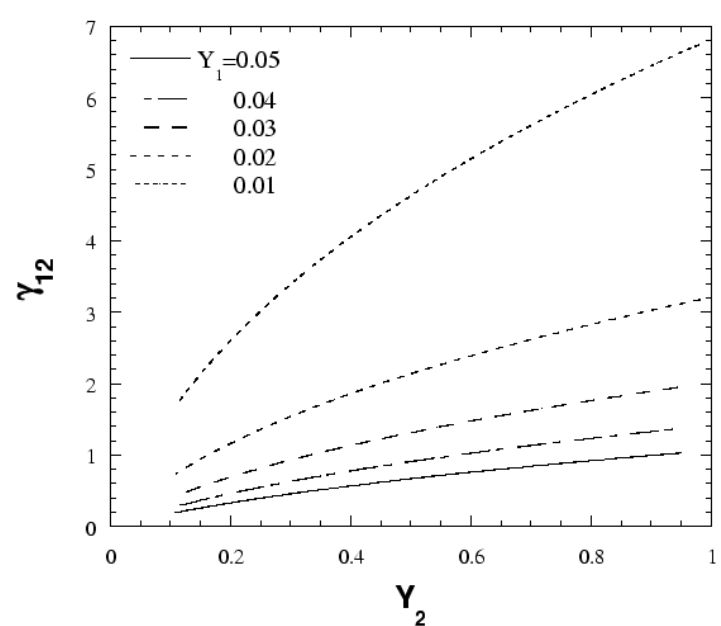

(b)

Figure 2. a) Shows $\gamma_{11}=d_{\theta}^{-} / q_{1} d_{1}$ as a function of the mass fraction of the n-heptane (fuel 2); b) shows $\gamma_{12}=\left(q_{2} d_{2} / q_{1} d_{1}\right)$ as a function of the mass fraction of the $\mathbf{n}$-heptane (fuel 2). 
The values of the parameters $d_{\theta}^{-} / q_{1} d_{1}$, defined as $\gamma_{11}$, and $\left(q_{2} d_{2} / q_{1} d_{1}\right)$, defined as $\gamma_{12}$, are determined by the leading order problem. The data used in this analysis are exhibited in figure (2). Even though they can be determined by any available commercial reacting flow code, in this analysis the data were obtained by a particular reacting flow code. ${ }^{12}$

\section{B. Solution of order $\varepsilon_{2}$}

The structure of layer 2 (see fig. 1), in which the oxidation of fuel 2 is performed, is described exactly as in Liñán's analysis. ${ }^{7}$ Recalling that the solutions of order of $\varepsilon_{1}$ are known at the condition $\xi_{1}=\xi_{1 f}$, then the expansion (1) has to be re-written as

$$
\begin{array}{rllll}
\theta & =\theta_{f}-\varepsilon_{1}\left(\Theta_{1 f}+\gamma_{1} \xi_{1 f}\right) / \delta_{1}^{1 / b_{1}} & -\varepsilon_{2}\left(\Theta_{2}+\gamma_{2} \xi_{2}+p\right) & +\cdots \\
y_{O}=0 & +\varepsilon_{1}\left(d_{1}+d_{2}\right) \Psi_{01 f} /\left(m_{1} \delta_{1}^{1 / b_{1}}\right) & +\varepsilon_{2} \Psi_{02} & +\cdots \\
y_{1}=0 & +\varepsilon_{1} d_{1} \Psi_{11 f} / m_{1} \delta_{1}^{1 / b_{1}} & +\varepsilon_{2} d_{1} \Psi_{12} / m_{2} & +\cdots \\
y_{2}=0 \quad+0 & +\varepsilon_{2} d_{2} \Psi_{22} / m_{2} & +\cdots \\
x & =x_{f}+\varepsilon_{1} \xi_{1 f} / m_{1} \delta_{1}^{1 / b_{1}} & +\varepsilon_{2}\left(\xi_{2}+p / \gamma_{2}\right) / m_{2} & +\cdots
\end{array}
$$

By taking Eq. (15) into the balance equations for species and energy and picking up the equations of the order of $\varepsilon_{2}$, one finds

$$
\begin{gathered}
\frac{d^{2}}{d \xi_{2}^{2}}\left(\Theta_{2}+\gamma_{2} \xi_{2}\right)=\frac{\bar{D} a_{2}}{\Gamma\left(\beta_{22}+1\right)} \Psi_{22}^{\beta_{22}} e^{-\left(\Theta_{2}+\gamma_{2} \xi_{2}+p\right)} \\
\frac{d^{2}}{d \xi_{2}^{2}} \Psi_{22}=\frac{\bar{D} a_{2}}{\Gamma\left(\beta_{22}+1\right)} \Psi_{22}^{\beta_{22}} e^{-\left(\Theta_{2}+\gamma_{2} \xi_{2}+p\right)}
\end{gathered}
$$

The properties of the problem is summarized in the parameter $\bar{D} a_{2}$ according to

$$
\bar{D} a_{2} \equiv \frac{\Gamma\left(\beta_{22}+1\right) q_{2} D a_{2}}{\theta_{f}^{* \alpha+\beta_{21}+\beta_{22}}}\left(\frac{\varepsilon_{1}\left(d_{1}+d_{2}\right) \Psi_{01 f}}{m_{1} \delta_{1}^{1 / b_{1}}}\right)^{\beta_{21}}\left(\frac{\varepsilon_{2}}{q_{2}}\right)^{\beta_{22}} \frac{\varepsilon_{2}}{m_{2}^{2}} \exp \left(-\theta_{a 2} / \theta_{f}^{*}\right)
$$

The boundary conditions for Eqs. (16) and (17), determined by the matching with the solution of order $\varepsilon_{1}$, are given by

$$
\begin{gathered}
\frac{d \Theta_{2}}{d \xi_{2}}=\frac{d \Psi_{22}}{d \xi_{2}}=0 \quad \xi_{2} \rightarrow-\infty \\
\frac{d \Theta_{2}}{d \xi_{2}}-1=\frac{d \Psi_{22}}{d \xi_{2}}-1=0 \quad \xi_{2} \rightarrow \infty
\end{gathered}
$$

Note that the definitions of $m_{2}$ and $\gamma_{2}$ are such that permit the derivative of $\Theta_{2}$ and $\Psi_{22}$ to be normalized for $\xi_{2} \rightarrow \infty$; recalling that, from the definition of $m_{2}$ and Eq. (8), $m_{2}=q_{2} d_{2}=d_{\theta}^{+*}-d_{\theta}^{-*}$. The values of $d_{\theta}^{-*}$ and $d_{\theta}^{-*}$ are the slope of temperature distribution at left side and at right side of $\xi_{1}=\xi_{1 f}$, respectively.

By combining Eqs. (16) and (17) and imposing the condition given by Eq. (18), the following relation is found

$$
\Psi_{22}-\Theta_{2}=0
$$


The choice for $p$ is such that the relation

$$
p=\ln \left(\bar{D} a_{2}\right)
$$

is satisfied.

The displacement $p / \gamma_{2}$ in the coordinate $\xi_{2}$, makes the problem (6), (7) and (14) dependent only on the value of $\gamma_{2}$. In order to match the inner and outer solutions, represented by the boundary conditions (18) and (19), it is necessary to impose

$$
p / \gamma_{2}=\lim _{\xi_{2} \rightarrow \infty}\left(\Psi_{22}-\xi_{2}\right)=\eta
$$

The solution of the problem represented by Eqs. (17) and (20), that satisfies the boundary conditions (18) and (19), determines the distribution of the n-heptane (fuel 2) inside the layer 2 and, at the same time, the value of the $\eta$.

An approximation for $\eta \gamma_{2}$ is given in Appendix B. It is considered the particular case $\beta_{21}=\beta_{22}=1$, that are the values presumed in all results presented subsenquently below. The value of the properties at the layer 2, $\Psi_{01 f}, \Psi_{11 f}$ and $\Theta_{1 f}$ at $\xi_{1}=\xi_{1 f}$, will be determined with the solution of Eq. (14) submitted to the boundaries conditions (6) and (7) and to the extra condition

$$
\Psi_{01 f}=\left[\Psi_{11 f}-\left(1+d_{2} / d_{1}\right) \xi_{1 f}\right] /\left(1+d_{2} / d_{1}\right)=\exp \left(\eta \gamma_{2}\right) / \tilde{D} a_{2}
$$

in which $\eta \gamma_{2}$ is estimated by Eq. (B-4) in Appendix B. The expression for $\tilde{D} a_{2}, \bar{D} a_{2} / \Psi_{01 f}$, is also shown in Appendix B.

Contrary to $\gamma_{11}$ and $\gamma_{12}$, which depend only on the properties of the leading order problem, $\gamma_{2}$ is a function of the properties of the $\varepsilon_{1}$ and $\varepsilon_{2}$ order problems by the dependence of the heat flux $d_{\theta}^{-*}$ to the oxygen side of the flame at the layer $2, \xi_{1}=\xi_{1 f}$. The dependence of $\gamma_{2}$ on those properties is summarized in figure (3).

Note that, $\gamma_{2}=d_{\theta}^{-*} / q_{2} d_{2}>1$ means that the heat flux from layer 2 is supported not only by the heat released by reaction 2 but also by that of the reaction 1 inside layer 1 .

The third condition, that specified the mass fraction of hydrogen (fuel 1) at the layer $2 \xi_{1}=\xi_{1 f}$ is expressed by (23). This condition is necessary to the integration of the problem represented by Eqs. (14), (6) and (7). It depends on the quantity $\eta \gamma_{2}$ that is given by an approximated expression displayed in figure (4)

\section{Results and Comments}

The integration of equation (14) together with the conditions (6), (7) and (23) determines the position of the layer $2, \xi_{1 f}$, and the hydrogen (fuel 1) leakage, $\Psi_{11-\infty}$, through the layer 1 without reacting with the 


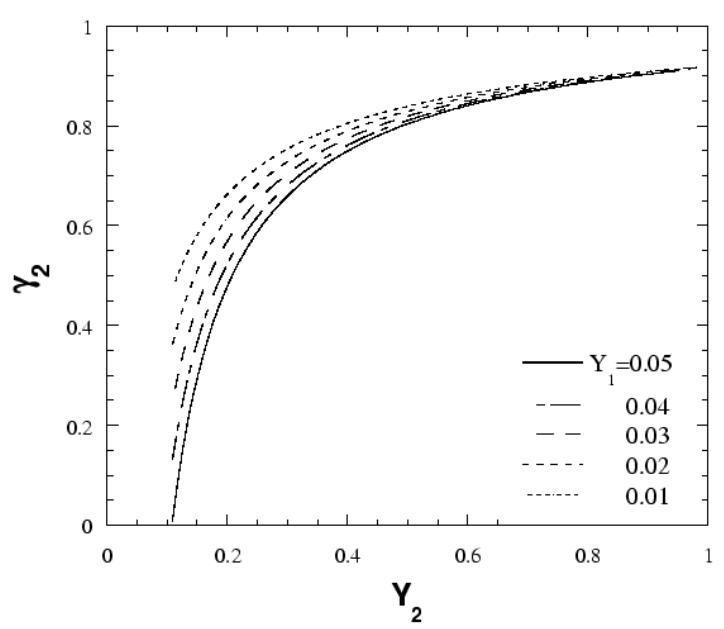

(a)

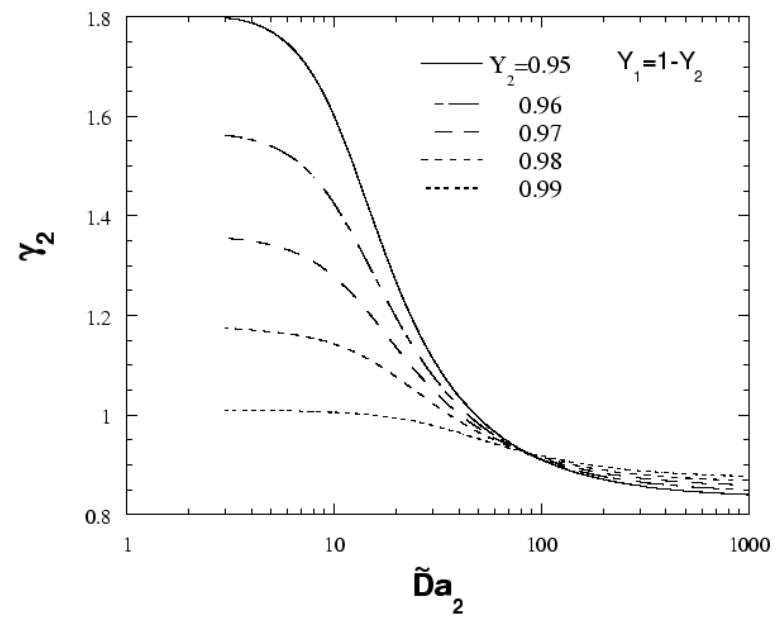

(b)

Figure 3. a) Parameter $\gamma_{2}=d_{\theta}^{-*} / q_{2} d_{2}$ as a function of the fuel composition for $\left.\tilde{D} a_{2}=100 ; \mathbf{b}\right)$ parameter $\gamma_{2}$ as a function of the Damköhler number $\tilde{D} a_{2}$, defined by (B-2).

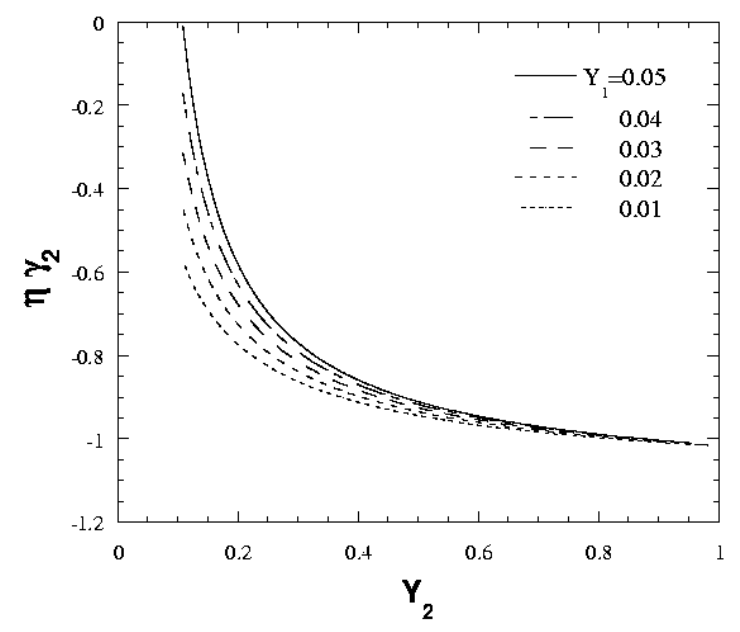

(a)

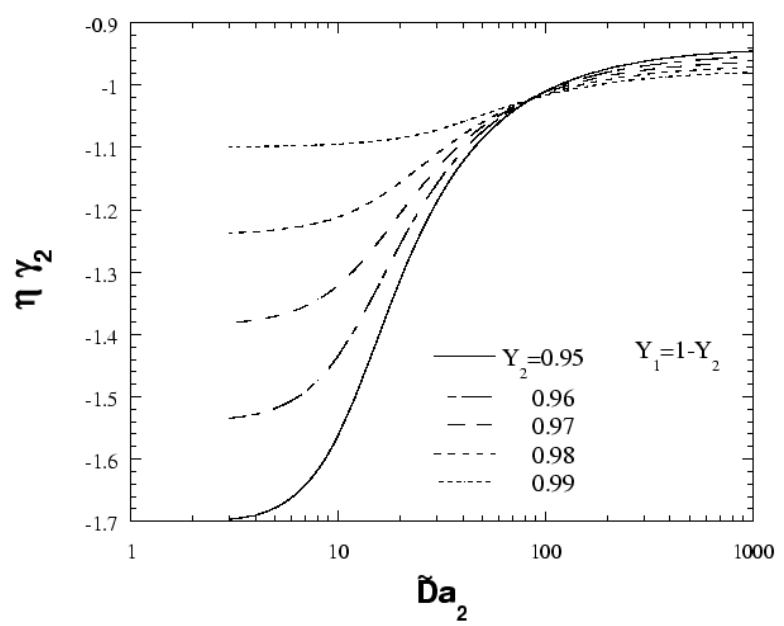

(b)

Figure 4. a) Shows $\eta \gamma_{2}$ as a function of the mass fraction of the $\mathbf{n}$-heptane (fuel 2) for $\tilde{D} a_{2}=100$, for five n-heptane (fuel 2) mass fraction; b) Shows $\eta \gamma_{2}$ as a function of Damköhler number $\tilde{D} a_{2}$, for five moistures . 
oxygen as a function of the modified Damköhler number $\delta_{1} /\left(d_{1} q_{1} / m_{1}\right)^{3}$ for given values of $\gamma_{11}$ and $\gamma_{12}$. The values of $\Psi_{11-\infty}$ are displayed in figure (5) for the fuel mixture composed by $Y_{1}=0.01$ and $Y_{2}=0.99$ and for the conditions inside the layer 2 such that $\tilde{D} a_{2}=100$.

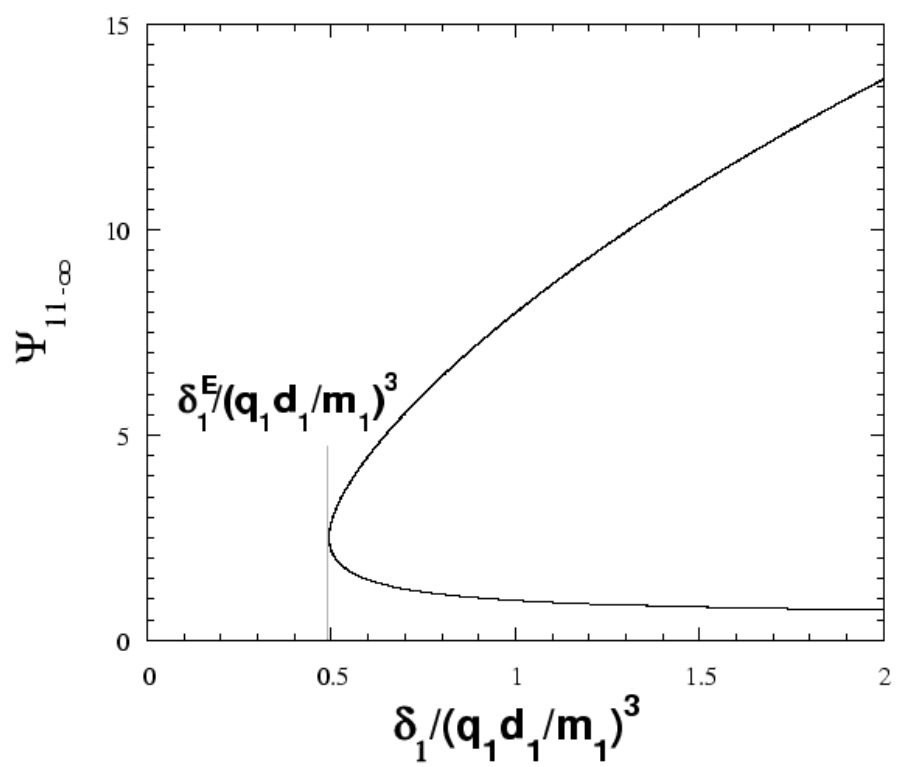

Figure 5. The hydrogen (fuel 1) mass fraction $\Psi_{11-\infty}$ leaking from the reaction zone 1 without reacting as a function of the modified Damköhler number $\delta_{1} /\left(d_{1} q_{1} / m_{1}\right)^{3}$

It is seen from fig. (5) that $\Psi_{11-\infty}$ has two values for each value of $\delta_{1} /\left(d_{1} q_{1} / m_{1}\right)^{3}$. The lower branch represents the stable solution and the upper branch the unstable solution. For $\delta_{1}^{E} /\left(d_{1} q_{1} / m_{1}\right)^{3}<0.5$, there is no a finite number of solutions and this limit points out the extinction condition of the flame.

The hydrogen does not have a large activation energy in order to permit good results from a large activation energy asymptotic analysis. Even so, that asymptotics was used because it produces qualitative results that will help the understanding of the future results. The hydrogen activation energy is assumed to be much larger than one but much smaller than that of the n-heptane $\left(\theta_{a 2} \gg \theta_{a 1} \gg 1\right)$.

The addition of hydrogen with n-heptane changes drastically the extinction condition of the n-heptane diffusion flame. Since hydrogen reaction has a smaller activation energy than that of the n-heptane reaction, the hydrogen reaction occurs in a zone (layer 1) thicker than the zone (layer 2) where the n-heptane reaction is present. As seen in figure (6), the position of layer $2, \xi_{1}-\xi_{1 f}=0$, characterized by the discontinuity of the derivative of the function $\Theta_{1}$, is inside layer 1. From this figure, it is also noted that the higher the hydrogen mass fraction is the thicker the layer 1 is. The thickness of the layer 1 can be visually determined by the deviation of the temperature profile from the frozen solution; $d \Theta_{1} / d \xi_{1}=-1$ for $-\infty<\xi_{1}<\xi_{1 f}$ and $d \Theta_{1} / d \xi_{1}=1$ for $\xi_{1 f}<\xi_{1}<\infty$.

12 of 21 


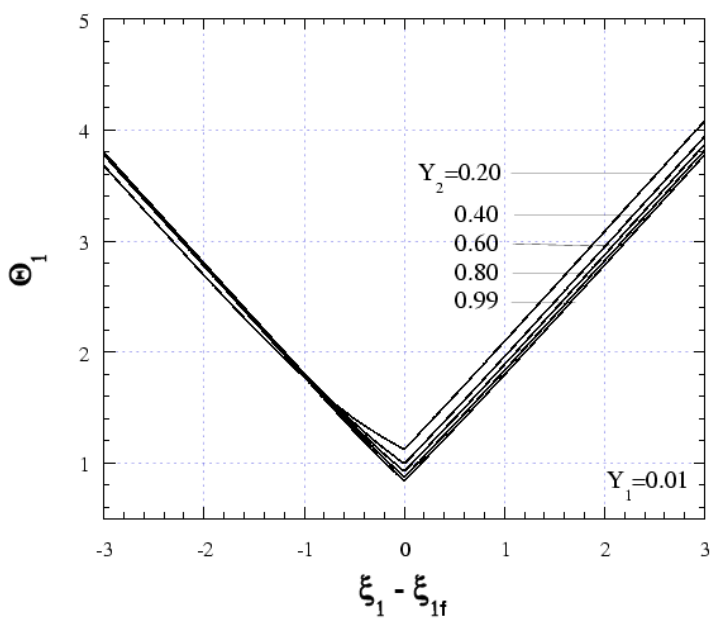

(a)

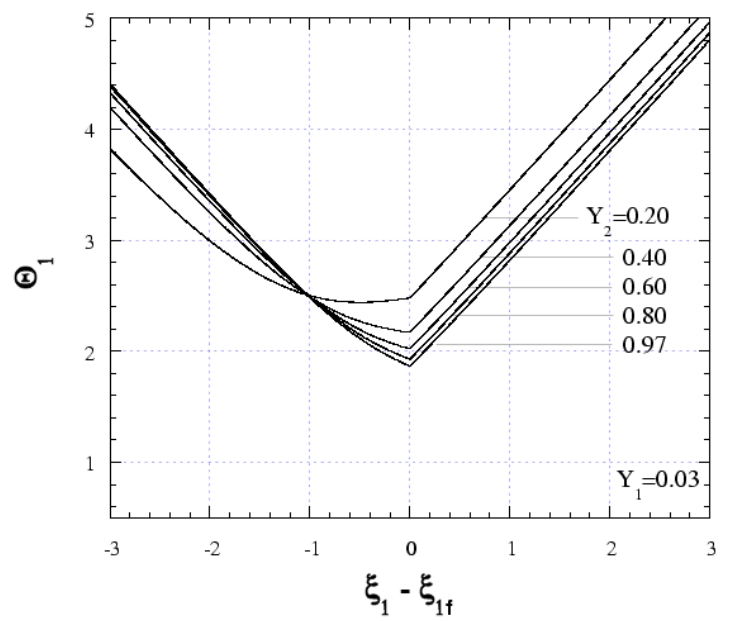

(c)

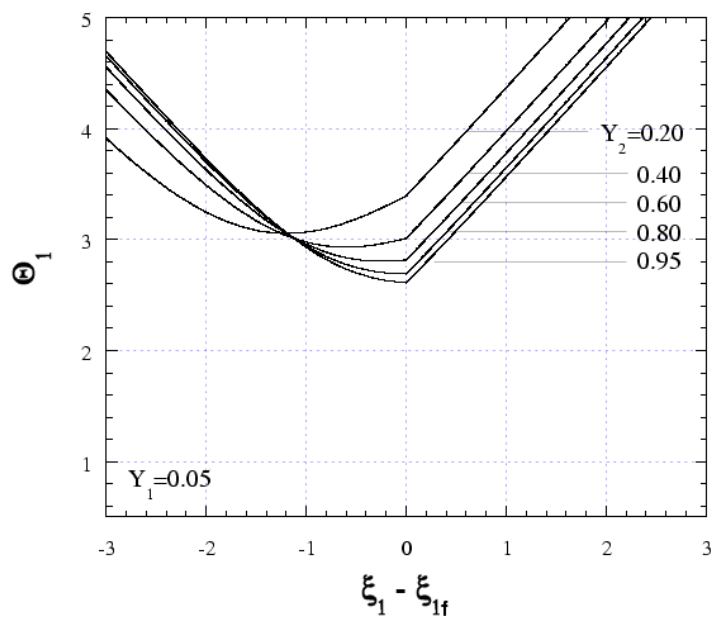

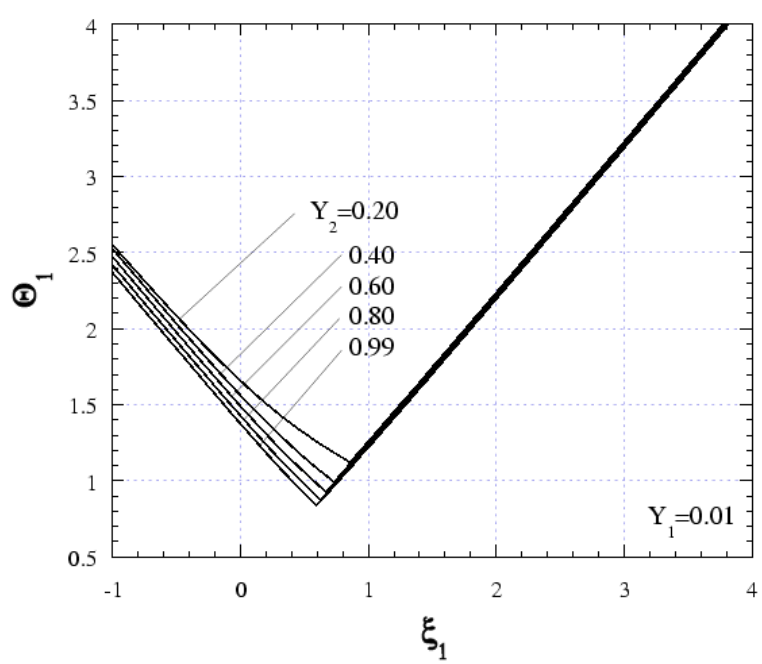

(b)

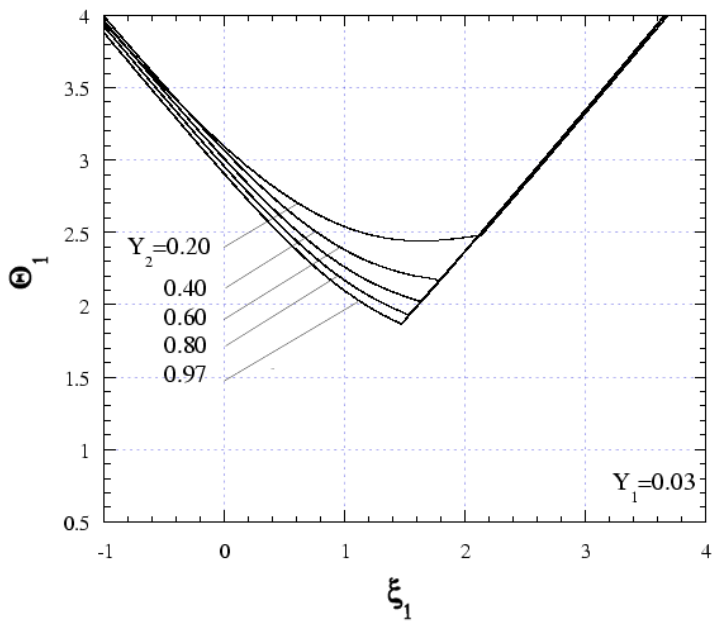

(d)

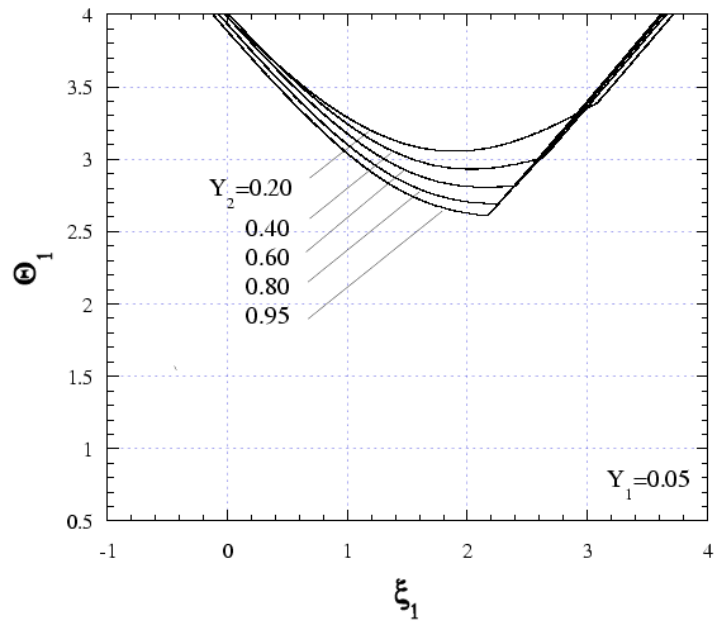

Figure 6. Temperature profile $\Theta_{1}$ inside the layer 13 for 21 three hydrogen mass fraction, $Y_{1}=0.01, \quad 0.03, \quad 0.05$. The plots at the right side are shifted $b_{1} \xi_{\text {f }}$ The $_{f}$ value of the Damköhler number $\tilde{D} a_{2}$ is 100. 
The temperature variation inside the layer 1 is expressed by $\left(\Theta_{1}+\gamma_{1} \xi_{1}\right) / \delta_{1}^{1 / b_{1}}$. Since the term $\gamma_{1} \xi_{1}$ is only a rotation, the variation of the temperature is collected in the term $\Theta_{1}$, henceforth called temperature.

Plots in the first column of figure (6) show the temperature profiles $\Theta_{1}$ as a function of the displaced spatial coordinate $\xi_{1}-\xi_{1 f}$. This form of presentation highlights easily the differences of the heat fluxes, $d \Theta_{1} /\left.d \xi_{1}\right|_{\xi_{1 f}^{-,+}}$, at both sides of layer 2 for different mixtures. By depending on the hydrogen mass fraction, the heat losses from layer 2 change even for $Y_{1}=0.01$ and $Y_{2}=0.20, d \Theta_{1} /\left.d \xi_{1}\right|_{\xi_{1 f}^{-}}<1$, as seen in figure (6.a). For larger hydrogen mass fraction, $Y_{1}=0.03$ and 0.05 , it can be observed that heat losses reduce strongly in one side of layer 2. Under this condition, the chemical reaction of the n-heptane becomes more difficult to extinguish. Also, it can be seen from these profiles and from the data for $\gamma_{2}=d_{\theta}^{-*} / q_{2} d_{2}$ in figure (3) that there are cases in which the heat flux $d_{\theta}^{-*}$ from layer 2 at the oxygen side is larger than the heat released by the n-heptane reaction for $\tilde{D} a_{2}<50$. This occurs because the layer 2 takes a position close to the flame border in the oxygen side and the heat flux from the layer 1 contributes to the heat flux from the layer 2 . Therefore, the temperature inside the layer 2 is an increasing function. This condition makes the n-heptane burning in the premixed-flame regime, because the n-heptane is totally consumed in the layer 2 , but not the oxygen.

Plots in the second column of figure (6) display the temperature profiles $\Theta_{1}$ as a function of the spatial coordinate $\xi_{1}$. From these plots, the displacement of the whole reaction zone, determined by the discontinuity in the first derivative, is specified for several compositions. As seen, the higher the mass fraction of the hydrogen $Y_{1}$ is, the thicker the layer 1 is and the deeper the position of layer $2, \xi_{1}=\xi_{1 f}$, in the fuel side of the flame. To understand this last result, it is necessary to include in the discussion the relation between the reactivity of the reaction 2 , given by the Damköhler number $\tilde{D} a_{2}$, and the oxygen mass fraction $\Psi_{01 f}$ at $\xi_{1}=\xi_{1 f}$. In the cases shown in figure (6), $\tilde{D} a_{2}$ is very high, $\tilde{D} a_{2}=100$, this means that the n-heptane (fuel 2) reaction takes place where oxygen mass fraction $\Psi_{01 f}$ is very low, of the order of $1 / \tilde{D} a_{2}$ according to Eq. (23). This condition is only found deep in the fuel side of the flame, $\xi_{1}>0$. Therefore, increasing the hydrogen mass fraction in the mixture, the layer 1 becomes thicker and the layer 2 is forced to stabilize deeper into the fuel side where the oxygen mass fraction is very low, $\Psi_{01} \sim 1 / \tilde{D} a_{2}$. In these cases, the layer 2 establishes a border $\left(\Psi_{01 f} \sim 0\right)$ for the layer 1 at the fuel side of the flame.

Note that, if the conditions are such that $1 / \tilde{D} a_{2} \sim \varepsilon_{2}$, the oxygen mass fraction at $\xi_{1}=\xi_{1 f}$ satisfies the following condition $\Psi_{01} \sim \varepsilon_{2}$. This condition is found for reactants consumption in the diffusion-flame burning regime. ${ }^{7}$ Thereby the burning of the n-heptane (fuel 2) changes from the premixed-flame regime to the diffusion-flame regime for $\Psi_{01 f} \sim \varepsilon_{2}$. The n-heptane diffusion-flame establishes a border for the flame in the fuel side. ${ }^{16}$

Also, from figure (6), it is seen that reducing the hydrogen (fuel 1) mass fraction in the mixture, the flame

14 of 21

American Institute of Aeronautics and Astronautics 
temperature, $\theta_{f}-\varepsilon_{1}\left(\Theta_{1 f}+\gamma_{1} \xi_{1 f}\right)$, increases because $\Theta_{1 f}$ decreases. This behavior is due to the increase of influence of the n-heptane (fuel 2) reaction on the total flame temperature.

The dependence of the temperature profiles on the modified Damköhler number $\tilde{D} a_{2}$ is displayed in figure (7). For $\tilde{D} a_{2}=100$, practically all hydrogen is consumed in a region located before the layer $2, \xi_{1}<\xi_{1 f}$. An indication of that is the small deviation of the temperature profile from the frozen solution, $d \Theta_{1} / d \xi_{1}=1$, in the region $\xi_{1}>\xi_{1 f}$. Also, it is seen that decreasing $\tilde{D} a_{2}$ the stabilization of layer 2 requires that the n-heptane (fuel 2) reaction takes place in a region with high oxygen mass fraction, that occurs in the oxygen side of the flame. For $\tilde{D} a_{2}=10$, practically all hydrogen is consumed in a region after the layer $2, \xi_{1}>\xi_{1 f}$. Note that for $\xi_{1}<\xi_{1 f}$, the temperature profile follows the frozen solution $d \Theta_{1} / d \xi_{1}=-1$.

Figure (7)b displays the conditions for the structure of the multicomponent fuel diffusion flames to be limited at one side of the flame; beyond that border there is no reaction. The flame presents a border in the oxygen side of the flame for small values of $\tilde{D} a_{2}(<10)$ and in the fuel side of the flame for large $\tilde{D} a_{2}$ $(>100)$.

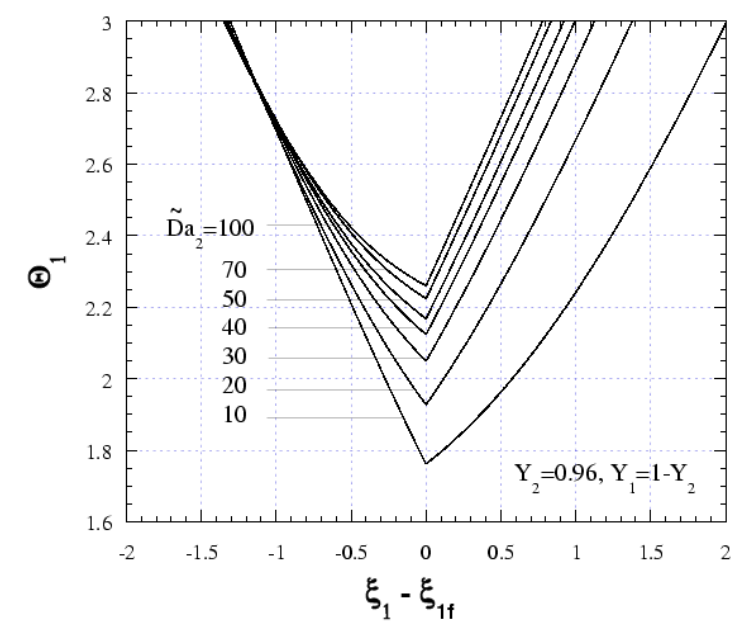

(a)

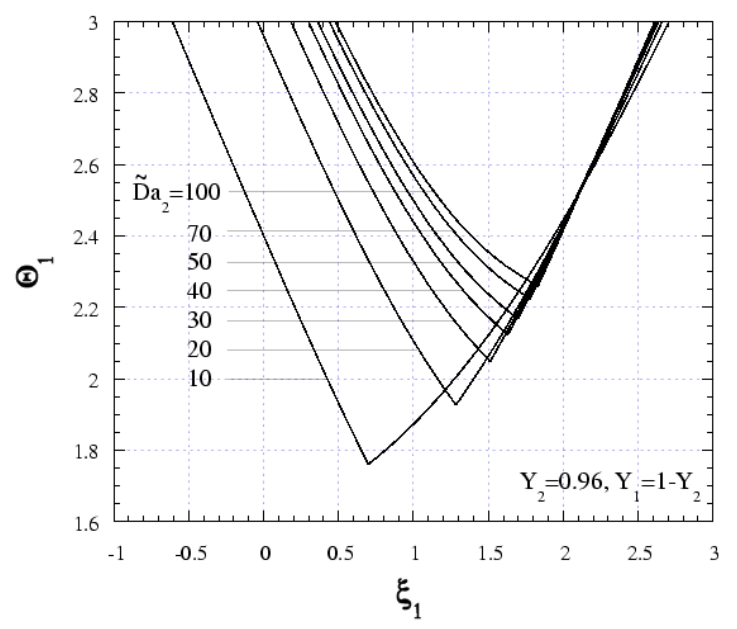

(b)

Figure 7. a) shows $\gamma_{11}=d_{\theta}^{-} / q_{1} d_{1}$ as a function of the mass fraction of the n-heptane (fuel 2); b) shows $\gamma_{12}=\left(q_{2} d_{2} / q_{1} d_{1}\right)$ as a function of the mass fraction of the $\mathbf{n}$-heptane (fuel 2).

The results depicted in Fig. (7) confirm the previous results, in that by increasing $\tilde{D} a_{2}$ the position of layer $2 \xi_{1}=\xi_{1 f}$ increases. The reason for that is because increasing the reaction rate of the n-heptane, the n-heptane reaction zone can stabilize in regions with lower oxygen mass fraction.

In the set of figures, (8),(9) and (10), other properties at layer 2 are presented and commented. In figures (8.a), (9.a), (10.a), the mass fractions of the hydrogen and oxygen at layer 2 and the position of layer 2, 
$\xi_{1}=\xi_{1 f}$, are displayed as a function of the fuel mixtures. As mentioned earlier, by increasing the influence of the hydrogen (fuel 1), that is done either by increasing the hydrogen mass fraction or by decreasing the n-heptane mass fraction in the mixture, the layer 2 stabilizes deeper into the fuel zone, $\xi_{1}>0$, where oxygen mass fraction $\Psi_{01 f}$ is small and the hydrogen mass fraction $\Psi_{11 f}$ is large, as seen in these figures.

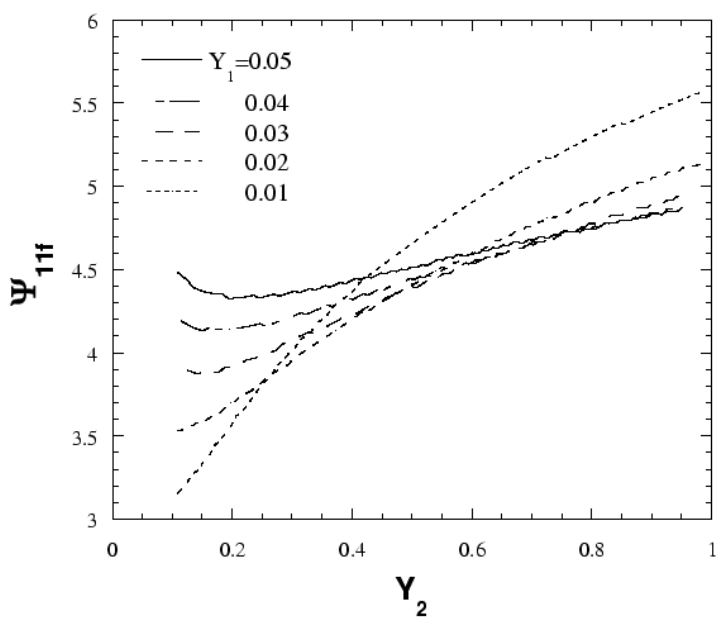

(a)

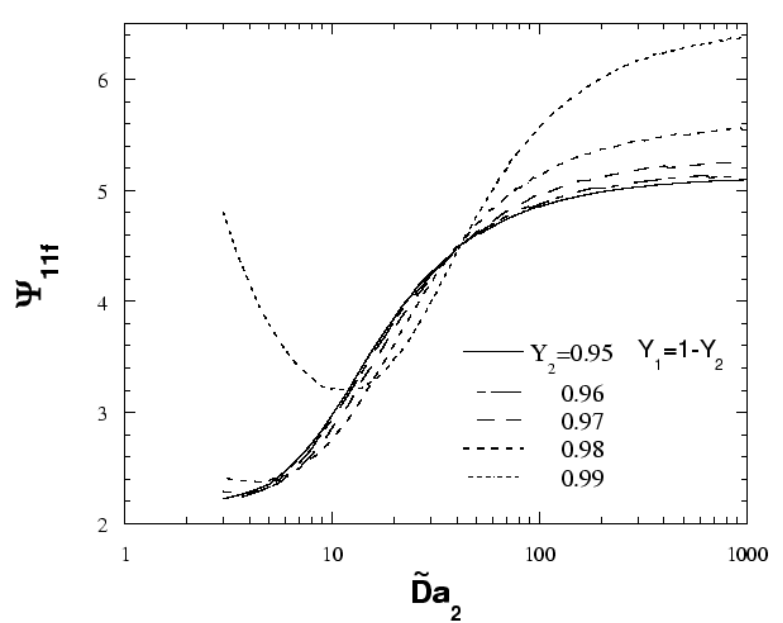

(b)

Figure 8. a) Hydrogen (fuel 1), mass fraction $\Psi_{11 f}$ at layer 2 as a function of mixture based on five hydrogen mass fraction, $Y_{1}=0.01-0.05$. These data are obtained assuming $\tilde{D} a_{2}=100$; b) hydrogen (fuel 1) mass fraction $\Psi_{11 f}$ at layer 2 as a function of $\tilde{D} a_{2}$, for five composition: $Y_{1}=0.01-0.05$ and $Y_{2}=1-Y_{1}$.

In figures (8.b), (9.b), (10.b), the mass fraction of the hydrogen and oxygen at layer 2 and the position of layer 2 are shown as a function of the $\tilde{D} a_{2}$. The variation of the $\tilde{D} a_{2}$ produces the most significant change in the layer 1 . It is easy to observe that for high values of $\tilde{D} a_{2}$, the layer 2 takes a position deep into the fuel side of the flame and, consequently, the oxygen mass fraction $\Psi_{01 f}$ goes to zero, according to $1 / \tilde{D} a_{2}$, and the hydrogen mass fraction to a fixed value. Under this conditions, not only the layer 1 but also layer 2 has a structure of diffusion flame. ${ }^{16}$ However, for $\tilde{D} a_{2}$ not too high, say less than 100 , the reaction 2 needs to takes place inside the layer 1 in the presence of high oxygen mass fraction in order to be stable. This condition means that the reaction 2 does not occur in stoichiometric conditions, but with an excess of oxygen. Then the internal structure of layer 2 is similar to that of the premixed flame, thereby the n-heptane (fuel 2) burns in the premixed-flame regime. ${ }^{7}$ For very low $\tilde{D} a_{2}, \tilde{D} a_{2}<10$, the oxygen leakage by layer 2 increases fast, the hydrogen mass fraction is low and the position $\xi_{1 f}$ goes to $\xi_{1}<0$, indicating a strong dependence of the reaction 2 on the oxygen..

Figure (9.b) reveals the conditions for the multicomponent fuel to be burned inside a single flame; $\tilde{D} a_{2}>3$. However, for conditions such that $\tilde{D} a_{2}<3$, the condition of single flame is not valid because 


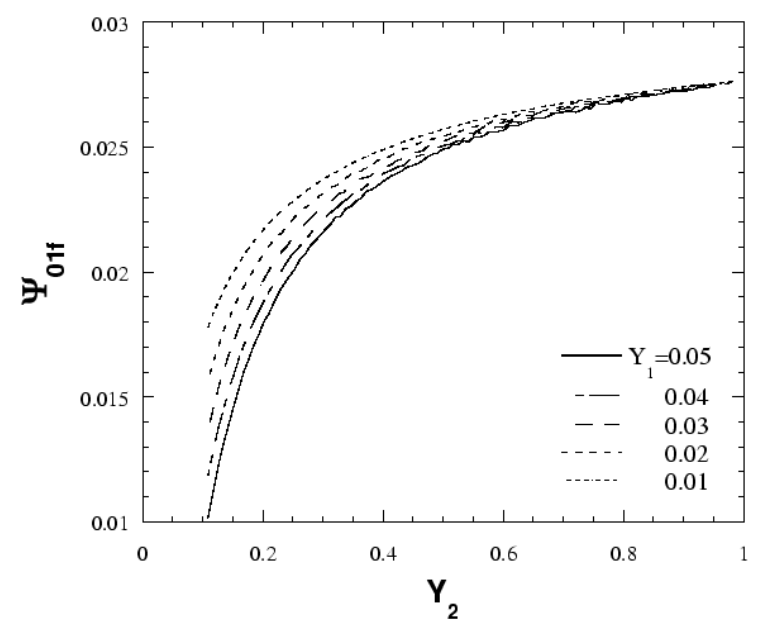

(a)

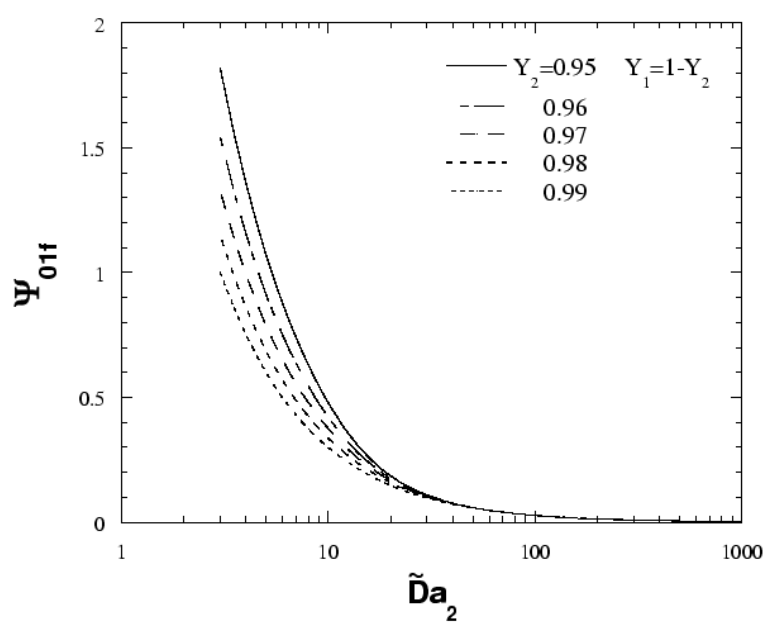

(b)

Figure 9. a) Oxygen mass fraction $\Psi_{01 f}$ at layer 2 as a function of mixture based on five hydrogen mass fraction, $Y_{1}=0.01-0.05$. These data are obtained assuming $\left.\tilde{D} a_{2}=100 ; \mathbf{b}\right)$ Oxygen mass fraction $\Psi_{01 f}$ at layer 2 as a function of $\tilde{D} a_{2}$, for five composition: $Y_{1}=0.01-0.05$ and $Y_{2}=1-Y_{1}$.

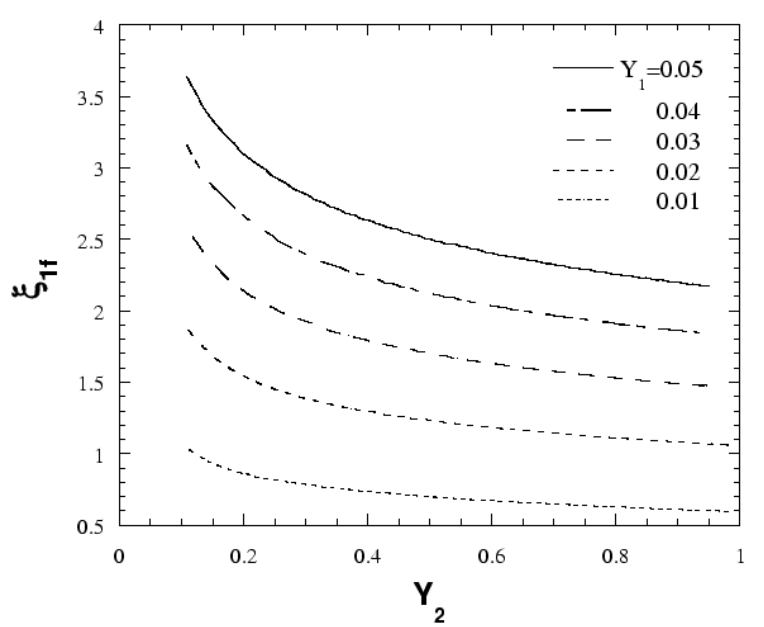

(a)

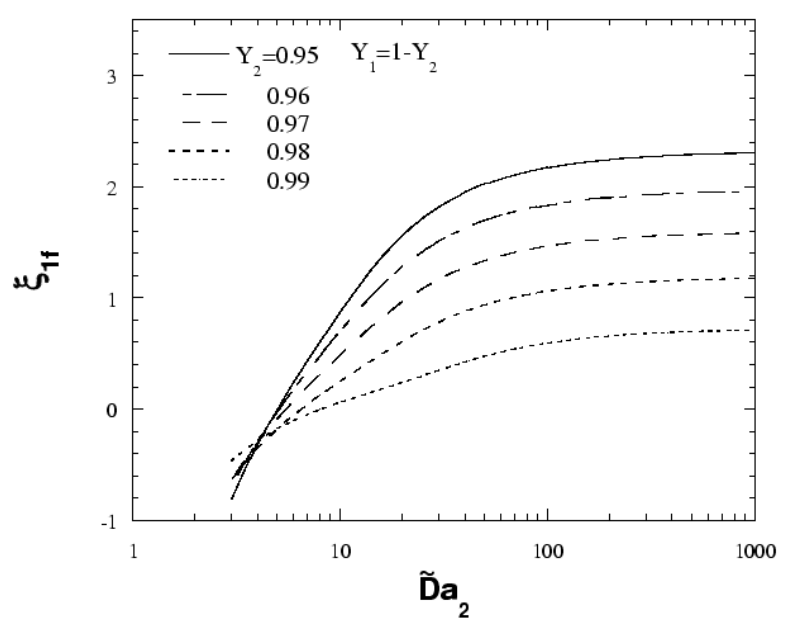

(b)

Figure 10. a) Position of layer $2 \xi_{1 f}$ inside layer 1 as a function of mixture based on five hydrogen mass fraction, $Y_{1}=0.01-0.05$. These data are obtained assuming $\left.\tilde{D} a_{2}=100 ; \mathbf{b}\right)$ Position of layer $2 \xi_{1 f}$ inside layer 1 as a function of $\tilde{D} a_{2}$, for five composition: $Y_{1}=0.01-0.05$ and $Y_{2}=1-Y_{1}$. 
the oxygen mass fraction at the layer 2 is very large, $\Psi_{01 f}>>1$. This condition makes the model invalid and indicates that the layer 2 is out of the layer 1 (hydrogen reaction zone). Therefore, for $\tilde{D} a_{2}<3$, the leading order problem must treat with two separate flames. As a result, the flame in the oxygen side will be a premixed-flame and the other flame in the fuel side will be a diffusion flame.

The curve corresponding to $Y_{2}=0.99$ and $Y_{1}=0.01$ in figure (8.b) indicates an increase in the hydrogen mass fraction at the layer 2. According to Fig. (10.b), the same combination of $Y_{1}$ and $Y_{2}$ does not result in an increase in the layer 2 position. These anomalous results can suggest a nonphysical condition in sense that it should be impossible a stable flame condition for $Y_{1}=0.01$ and $\tilde{D} a_{2}<10$.

In what follows, it is important to know the limit condition for the hydrogen reaction in the diffusionflame structure, since the addition of the hydrogen (fuel 1) conducts to a more stable reaction for n-heptane, That limit determines the extinction of the flame with a configuration established by the combination of diffusion-flame and premixed-flame structures.

Figure (11.a) shows the influence of the hydrogen and n-heptane mass fractions on the stabilization of the flame. The modified Damköhler number of the reaction 1 in the extinction condition, $\delta_{1}^{E} /\left(q_{2} d_{2} / m_{1}\right)^{3}$, is small for either higher hydrogen mass fraction or lower n-heptane mass fraction. This means that the flame is more stable under these conditions.

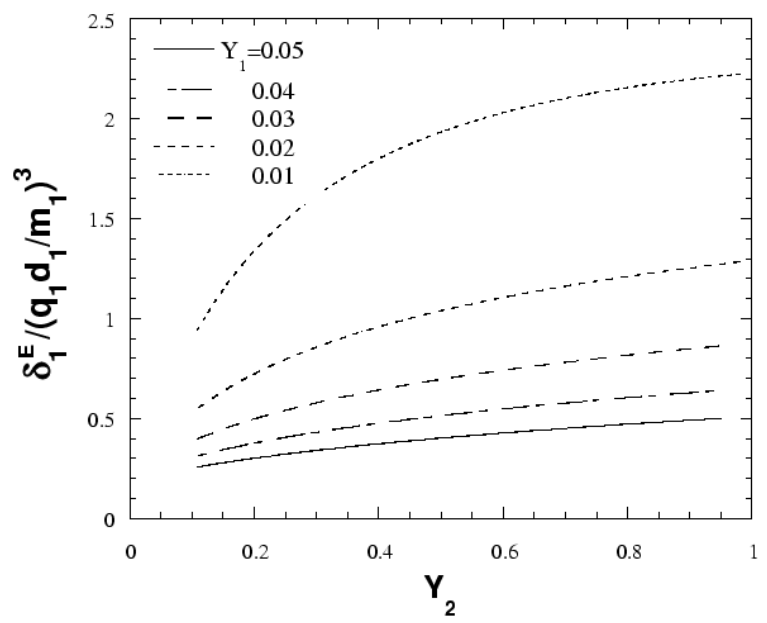

(a)

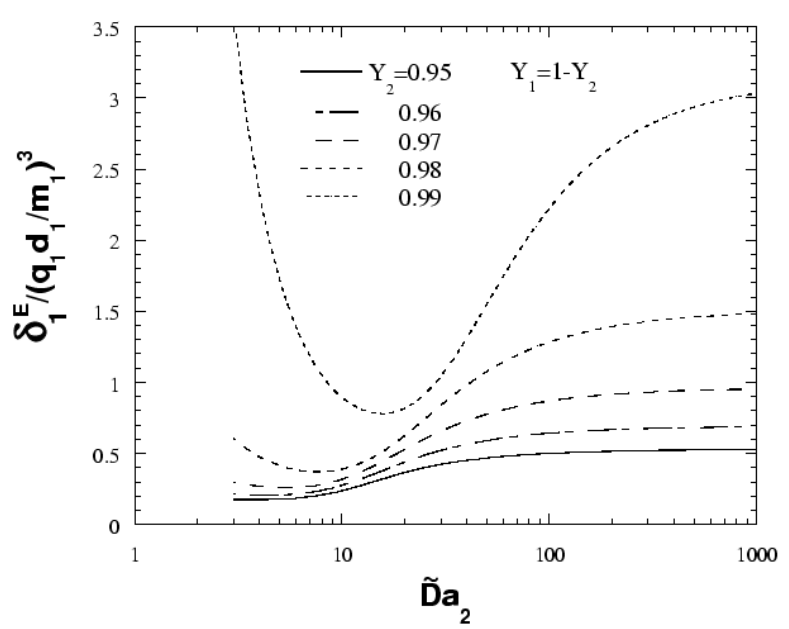

(b)

Figure 11. a)Shows the modified $\delta_{1}^{E} /\left(q_{2} d_{2} / m_{1}\right)^{3}$ as a function of the n-heptane (fuel 2) mass fraction for several values for hydrogen (fuel 1) mass fraction and $\left.\tilde{D} a_{2}=100 ; \mathbf{b}\right)$ shows the modified $\delta_{1}^{E} /\left(q_{2} d_{2} / m_{1}\right)^{3}$ as a function of the hydrogen (fuel 2) reactivity $\tilde{D} a_{2}$ for five compositions of hydrogen and n-heptane.

Figure (11.b) displays the influence of the reactivity of the reaction of n-heptane (fuel 2), represented by $\tilde{D} a_{2}$, on the stabilization of the layer 1 structure. Alternatively, figure (11.b) can also show implicitly the 
dependence of the layer 1 on the layer 2 position $\xi_{1}=\xi_{i f}$.

Contrary to the initial ideas (less oxygen leakage, or for the higher $\tilde{D} a_{2}$, the more stable the flame is), the results revealed that increasing $\tilde{D} a_{2}$ the stability of the layer 1 is reduced. These data reveal the importance of the heat losses from the flame in comparison to the oxygen leakage. To understand the results, it is essential to recall that the heat flux from the flame to the oxygen side of the flame is larger than that to the fuel side of the flame. ${ }^{17}$ Therefore, the flame is more stable for the conditions which make the layer 2 to take place closer to the oxygen side of the flame. This is because part of the heat flux from the layer 1 is supplied by the heat released in layer 2 . In addition, the importance of the layer 2 position on the flame stability can also be seen from the values for $\gamma_{2}$. For $\tilde{D} a_{2}<50, \gamma_{2}>1$ (figure 3) indicates that all heat released inside the layer 2 is transfer to the oxygen side of the flame. This makes the layer 1 structure more stable by reducing $\tilde{D} a_{2}$.

An opposite situation occurs for $\tilde{D} a_{2}>100$. In these cases, besides the layer 2 takes a fixed position in the fuel side establishing a border for the flame, not all heat released in it is transferred to frozen zone, as indicated by $\left(1-\gamma_{2}\right)<1$, figure (3). Then, an increase in the $\tilde{D} a_{2}$ leads to a less stable hydrogen reaction: the higher $\tilde{D} a_{2}$ is, the higher $\delta_{1}^{E} /\left(q_{2} d_{2} / m_{1}\right)^{3}$ is.

As mentioned earlier, the case represented by $Y_{1}=0.01$ and $Y_{2}=0.99$, as commented before, can represent an nonphysical flame condition because the results for $\Psi_{11 f}$ does not agree with that for $\xi_{1 f}$.

\section{Conclusions}

The addition of hydrogen in n-heptane diffusion flames changes drastically the flame structure and leads to more stable flames. A reason for that is because the main control of the flame stability is transfered from the n-heptane reaction to the hydrogen reaction. However, the n-heptane reaction contributes strongly to the hydrogen reaction. It was found that as the Damköhler number for the n-heptane reaction is in the range $10<\tilde{D} a_{2}<100$, the oxygen leakage from the n-heptane reaction zone is of the order of magnitude $\varepsilon_{1}$ and the $\mathrm{n}$-heptane reaction zone is inside the hydrogen reaction zone. Thereby, n-heptane reaction supplies heat for the hydrogen reaction.

Since the n-heptane reaction occurs under the condition of excess of oxygen, it consumption follows the premixed-flame regime. Thus, the whole flame structure is a combination of two types: a diffusion-flame structure and a premixed-flame structure. The former is imposed by the hydrogen and the latter is imposed by the n-heptane consumption.

For large $\tilde{D} a_{2}(>100)$, the thin n-heptane reaction zone establishes a border for the thick hydrogen reaction zone in the fuel side of the flame. However, for small $\tilde{D} a_{2}(<10)$, the thin n-heptane reaction zone

19 of 21

American Institute of Aeronautics and Astronautics 
establishes a border for the thick hydrogen reaction zone in the oxygen side of the flame, but this border is for the thermal problem, $d \Theta_{1} / d \xi_{1}=-1$, in the region $\xi_{1}<\xi_{1 f}$. As noted, this model is not valid for $\tilde{D} a_{2}<3$ because the oxygen mass fraction in the layer $2 \Psi_{01 f}$ is larger than unity; an indication of two separate flames in the leading order problem.

For the distinguished limit $1 / \tilde{D} a_{2} \sim \varepsilon_{2}$, the oxygen and n-heptane mass fractions have the same order of magnitude inside the thin n-heptane reaction zone, thereby the n-heptane consumption follows the diffusion-flame regime. The whole flame structure becomes a combination of two diffusion-flame structures. Furthermore, for $1 / \tilde{D} a_{2}<<\varepsilon_{2}$, the n-heptane consumption change to the rich-premixed-flame regime.

Acknowledgments This work was in part supported by the Conselho Nacional de Desenvolvimento Científico e Tecnológico - CNPq under the Grant 302801/03-0 and by the Fundação de Ampara à Pesquisa do Estado de São Paulo - FAPESP under the Grants 00/08997-4 and 00/08998-0.

\section{References}

\footnotetext{
${ }^{1}$ Hamins, A. and Seshadri, K., "Structure of Counterflow Diffusion Flames Burning Multicomponent Fuels," Proc. Combust. Instit., Vol. 20, 1984, pp. 1905-1913.

${ }^{2}$ Fachini, F. F., "Multicomponent Fuel Diffusion Flames: Flame Structure for a Single Diffusion-Flame Burning Regime," under consideration for publication, 2004.

${ }^{3}$ Krishnamurthy, L., Williams, F. A., and Seshadri, K., "Asymptotic Theory of Diffusion-Flame Extinction in the Stagnation-Point Boundary Layer," Combust. Flame, Vol. 26, 1976, pp. 363-378.

${ }^{4}$ Hamins, A. and Seshadri, K., "Prediction of Overall Chemical Kinetic Rate Parameters near Extinction for Diffusion Flame Burning Multicomponent Fuels," Combust. Sci. Technol., Vol. 38, 1984, pp. 89-103.

${ }^{5}$ Hamins, A. and Seshadri, K., "The Influence of Alcohol on the Combustion of Hydrocarbon Fuels in Diffusion Flames," Combust. Flame, Vol. 64, 1986, pp. 43-54.

${ }^{6}$ Hamins, A. and Seshadri, K., "The Structure of Diffusion Flames Burning Pure, Binary, and Ternary Solutions of Methanol, Heptane, and Toluene," Combust. Flame, Vol. 68, 1987, pp. 295-307.

${ }^{7}$ Liñán, A., "The Asymptotic Structure of CounterFlow Diffusion Flame for Large Activation Energy," Acta Astronautica, Vol. 1, 1974, pp. 1007-1039.

${ }^{8}$ Hubbard, G. L. and Tien, C. L., "Infrared Mean Absorption Coefficients of Luminous Flames and Smoke," Journal of Heat Transfer, Vol. 100, 1978, pp. 235-239.

${ }^{9}$ Rightley, M. L. and Williams, F. A., "Structures of CO Diffusion Flames Near Extinction," Combust. Sci. Technol., Vol. 125, 1997, pp. 181-200.

${ }^{10}$ Fachini, F. F., Liñán, A., and Williams, F. A., "Theory of Flame Histories in Droplet Combustion at Small Stoichiometric Fuel-Air Ratios," AIAA Journal, Vol. 37, 1999, pp. 1426-1435.

${ }^{11}$ Fachini, F. F. and Seshadri, K., "Rate-Ratio Asymptotic Analysis of Nonpremixed n-Heptane Flames," Combust. Sci. Technol., Vol. 175, 2003, pp. 125-155.

${ }^{12}$ Fachini, F. F., "Multicomponent Fuel Diffusion Flames: A Generalized Shvab-Zel'dovich Formulation," to be submitted, 2004.

${ }^{13}$ Seshadri, K. and Williams, F., "Laminar Flow Between Parallel Plates with Injection of a Reactant at a High Reynolds Number," Int. J. Heat Mass Transfer, Vol. 21, 1978, pp. 251-253.

${ }^{14}$ Liñán, A., "The Structure of Diffusion Flames," Fluid Dynamical Aspects of Combustion Theory, Longman Scientific and Technical (Pitman Research Notes in Mathematics, No. 223), Harlow, U.K., 1991, pp. 11-29.

${ }^{15}$ Fachini, F. F., "An Analytical Solution for the Quasi-Steady Droplet Combustion," Combust. Flame, Vol. 116, 1999, pp. 302-306.

${ }^{16}$ Fachini, F., "Multicomponent-Fuel Diffusion Flames: Flame Structure for Coupled Diffusion-Flame Burning Regimes," to be submitted, 2004.

${ }^{17}$ Peters, N., "Local Quenching Due to Flame Stretch and Non-Premixed Turbulent Combution," Combust. Sci. Tech., Vol. 30, 1983, pp. 1-17.
}

20 of 21 


\section{A. Appendix}

The value of the $d_{\theta}^{-*}$, the temperature slope at $\xi_{1}=\xi_{1 f}^{-}$, is expressed by

$$
d_{\theta}^{-*}=\left.\frac{d}{d \xi_{1}}\left[m_{1}\left(\Theta_{1}+\gamma_{1} \xi_{1}\right)\right]\right|_{\xi_{1}=\xi_{1 f}^{-}}=m_{1} \Theta_{1 f^{-}}^{\prime}+m_{1} \gamma_{1}
$$

Since $\gamma_{1}=1-d_{\theta}^{-} / m_{1}$ and $m_{1} \gamma_{1}=m_{1}-d_{\theta}^{-}$, then

$$
d_{\theta}^{-*}=m_{1} \Theta_{1 f^{-}}^{\prime}+\left(m_{1}-d_{\theta}^{-}\right)
$$

From Eq. (12), Eq. (A-2) can be written in terms of $\Psi_{21}$ as following

$$
d_{\theta}^{-*}=q_{1} d_{1} \Psi_{11 f}^{\prime}-d_{\theta}^{-}
$$

\section{B. Appendix}

From the definition of $\bar{D} a_{1}$ and Eqs. (21) and (22), the translation on the coordinate given by $p$ can be related with physical and chemical properties of the problem through

$$
\frac{\Gamma\left(\beta_{12}+1\right) q_{1} D a_{1}}{\theta_{f}^{* \alpha+\beta_{11}+\beta_{12}}}\left(\frac{\varepsilon_{1}\left(d_{1}+d_{2}\right) \Psi_{01 f}}{m_{1} \delta_{1}^{1 / b_{1}}}\right)^{\beta_{11}}\left(\frac{\varepsilon_{2}}{q_{1}}\right)^{\beta_{12}} \frac{\varepsilon_{2}}{m_{2}^{2}} \exp \left(-\theta_{a 1} / \theta_{f}^{*}\right)=\exp \left(\eta \gamma_{2}\right)
$$

Since the value of the $D a_{1}$ is in a large range, it is possible to work with fixed value of

$$
\tilde{D} a_{1} \equiv \frac{\Gamma\left(\beta_{12}+1\right) q_{1} D a_{1}}{\theta_{f}^{* \alpha+\beta_{11}+\beta_{12}}}\left(\frac{\varepsilon_{1}\left(d_{1}+d_{2}\right)}{m_{1} \delta_{1}^{1 / b_{1}}}\right)^{\beta_{11}}\left(\frac{\varepsilon_{2}}{q_{1}}\right)^{\beta_{12}} \frac{\varepsilon_{2}}{m_{2}^{2}} \exp \left(-\theta_{a 1} / \theta_{f}^{*}\right),
$$

then the oxygen mass fraction at the layer $2, \xi_{1}=\xi_{1 f}$ can be specified according to

$$
\Psi_{01 f}^{\beta_{11}}=\exp \left(\eta \gamma_{2}\right) / \tilde{D} a_{1}
$$

After the problem represented by Eqs. (6), (7) and (14) is solved satisfying the condition Eq. (B-3), the value of $\Theta_{1 f}$ is determined by Eq. (12) and the value of the $D a_{1}$ is found by Eq. (B-2).

In this work, it is considered the particular case $\beta_{11}=\beta_{12}=1$, because there are approximations for $\eta \gamma_{2}$ ( Liñán, 1974) as

$$
-\eta \gamma_{2}=\left\{\begin{array}{ccc}
-1.344 \gamma_{2}-4 \gamma_{2}^{2}\left(1-\gamma_{2}\right) /\left(1-2 \gamma_{2}\right)- & \\
3 \gamma_{2}^{3}-\ln \left(1-4 \gamma_{2}^{2}\right) & \text { for } & -0.5<\gamma_{2}<0.2 \\
-\ln \left(0.6307 \gamma_{2}^{2}+1.344 \gamma_{2}+1\right) & \text { for } & 0 \leq \gamma_{2}
\end{array}\right.
$$

